study has convincingly demonstrated that thin-strand pericytes have a 'tense relationship' with capillaries, squeezing out new questions and relaxing the barriers for new debate.

Adam Institoris and Grant R. Gordon (D) $₫$ Hotchkiss Brain Institute, Department of Physiology and Pharmacology, Cumming School of Medicine, University of Calgary, Calgary, Alberta, Canada.

$凶_{e-\text { mail: gordong@ucalgary.ca }}$
Published online: 21 April 2021

https://doi.org/10.1038/s41593-021-00853-1

References

1. Pough, F. H. American Zoologist 20, 173-185 (1980).

2. Gould, I. G., Tsai, P., Kleinfeld, D. \& Linninger, A. J. Cereb. Blood Flow Metab. 37, 52-68 (2017).

3. Attwell, D., Mishra, A., Hall, C. N., O'Farrell, F. M. \& Dalkara, T. I. Cereb. Blood Flow Metab. 36, 451-455 (2016).

Hall, C. N. et al. Nature 508, 55-60 (2014).

5. Kisler, K. et al. Nat. Neurosci. 20, 406-416 (2017).

6. Hill, R. A. et al. Neuron 87, 95-110 (2015).

7. Fernández-Klett, F., Offenhauser, N., Dirnagl, U., Priller, J. \&

Lindauer, U. Proc. Natl Acad. Sci. USA 107, 22290-22295 (2010).
8. Hartmann, D. A. et al. Nat. Neurosci. https://doi.org/10.1038/ s41593-020-00793-2 (2021).

9. Kureli, G. et al. Exp. Neurol. 332, 113392 (2020).

10. Gutiérrez-Jiménez, E. et al. J. Cereb. Blood Flow Metab. 38 , 290-303 (2018)

11. Watson, A. N. et al. J. Cereb. Blood Flow Metab. 40, 2387-2400 (2020).

12. Alarcon-Martinez, L. et al. Nature 585, 91-95 (2020).

13. Yemisci, M. et al. Nat Med 15, 1031-1037 (2009).

14. Alarcon-Martinez, L. et al. Acta Neuropathol. Commun. 7, 134 (2019).

15. Nortley, R. et al. Science 365, eaav9518 (2019).

Competing interests

The authors declare no competing interests.

\title{
To catch a memory through covert ops
}

\author{
Disrupting reconsolidation of the maladaptive memories underlying post-traumatic stress disorder (PTSD) could \\ be transformative for treatment. However, patients cannot undergo the direct re-exposure to trauma-cues used \\ to induce reconsolidation in animal studies. Ressler and colleagues report 'covert' memory reactivation in rats, \\ bolstering hopes for translation of reconsolidation-based interventions.
}

\section{Amy L. Milton}

$\mathrm{M}$ ental health disorders are a major global health problem, estimated to affect 792 million people worldwide ${ }^{1}$. One particularly distressing and debilitating mental health disorder, predicted to increase in prevalence following the global COVID-19 pandemic ${ }^{2}$, is post-traumatic stress disorder (PTSD) ${ }^{3}$. PTSD is diagnosed following exposure to a traumatic event (for example, a near-death experience in an intensive care unit) and is characterized by intrusive thoughts of the trauma, flashbacks, nightmares, hyper-reactivity, and extreme psychological distress in response to trauma-related cues. A major theory of PTSD suggests that the persistence and pervasiveness of the disorder is due to the formation of maladaptive fear memories that come to dominate cognition and behavior ${ }^{4}$. Reducing the impact of these maladaptive memories has been a major treatment focus, leading to the development of therapies such as prolonged exposure therapy. However, prolonged exposure is based upon the psychological process of extinction and, despite innovative adaptations to the treatment protocol ${ }^{5}$, the maladaptive fear memory still returns in approximately half of patients ${ }^{6}$. Further treatment innovation is clearly needed.

One potential new approach to treating PTSD is to try to disrupt the original fear memory itself. This could be through targeting the initial consolidation of the memory, but for a variety of reasonsincluding the practical difficulties of reaching those affected by trauma within the 4-6-h consolidation 'window'-many investigators have instead focused on targeting fear memory reconsolidation. Reconsolidation is the process by which memories can become modifiable under certain conditions of retrieval $^{7}$, and although there appear to be specific 'boundary conditions' controlling whether reconsolidation occurs, it has been reported that even old, well-established memories can become susceptible to disruption with amnestic agents following an appropriate 'memory reactivation' session ${ }^{8}$. However, reconsolidation appears to be highly specific to the memories that are reactivated. For example, in rats, when two cues are paired with an aversive footshock outcome, reactivation of one cue induces that cue-footshock association to become unstable, but it leaves the non-reactivated cue-footshock outcome intact ${ }^{9}$. This could present a challenge for the translation of reconsolidation-based therapies to the clinic, where reactivation of the memory involves indirect re-exposure (i.e., only some of the cues associated with the trauma are presented) or even imaginal exposure (where the person is asked to imagine the trauma or trauma-related cues). Thus, for reconsolidation purposes the crucial question is: is it possible to cause a memory to become unstable through indirect re-exposure? New data in Nature Neuroscience from Ressler and colleagues ${ }^{10}$ suggest that it is.

Using a combination of sophisticated behavioral approaches and engram tagging and manipulation techniques in rats, Ressler and colleagues ${ }^{10}$ demonstrate that it is possible to 'covertly' reactivate and attenuate a fear memory. To investigate covert reactivation, they used a backward fear conditioning procedure, which they contrasted to the more usual 'forward' auditory fear conditioning (Fig. 1). In forward auditory fear conditioning, rats are trained to associate an auditory cue, which acts as a Pavlovian conditioned stimulus, with an electric footshock outcome. The cue develops both predictive propertiespredicting the delivery of the shock-and affective properties, becoming a fearful stimulus in its own right. Forward fear conditioning may take place in a specific context, but often rats more strongly associate the cue with shock than the context, because the cue is a better predictor. By contrast, in backward conditioning, the cue is presented after the shock delivery. Consequently, the cue does not predict the shock itself; rather, the context is the better predictor in this situation. However, subsequent presentation of the cue serves to indirectly reactivate the memory of the context and thereby (potentially) the context-shock association. This behavior therefore provides an excellent opportunity 
a Forward conditioning

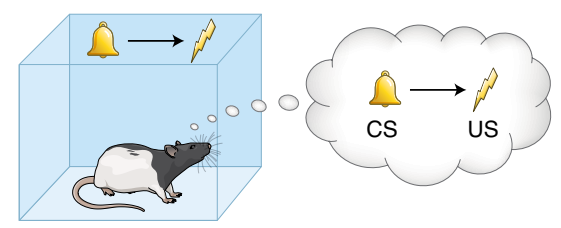

b Backward conditioning

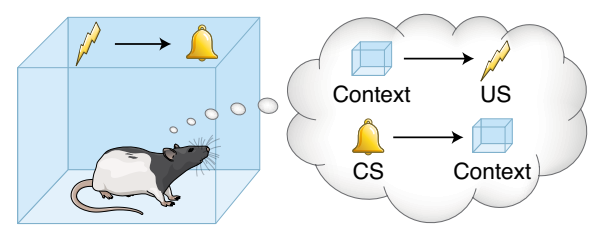

C Memory reactivation

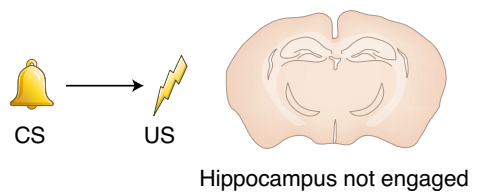

d Memory reactivation

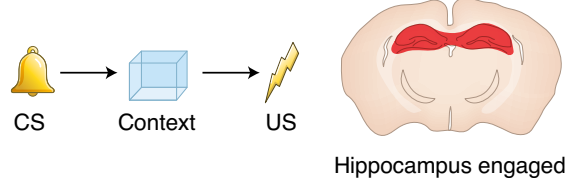

e 'Covert' capture and attenuation

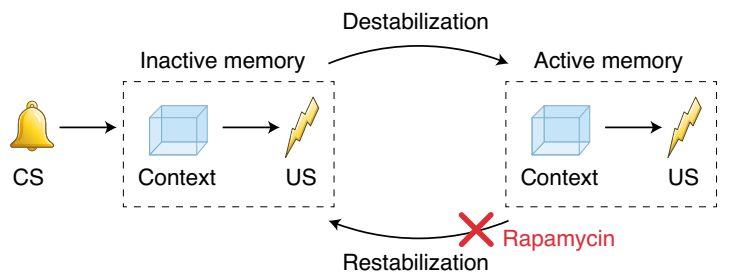

Fig. 1| Backward conditioning as a method for demonstrating covert capture of a memory. a,b, In both forward (a) and backward (b) fear conditioning, rats are trained to associate a conditioned stimulus (CS) with an unconditioned stimulus (US) in a specific context. In forward conditioning, the CS predicts the delivery of the footshock leading the rat to form a CS $\rightarrow$ US association (shown in thought bubble). In backward conditioning, the CS follows the US, causing the rat to form two associations; one of the context $\rightarrow U S$ and another of the CS $\rightarrow$ context (shown in thought bubble). c, When a rat that has undergone forward conditioning is presented with the CS, it reactivates the memory of the CS $\rightarrow$ US association in a manner that is largely independent of the hippocampus. $\mathbf{d}_{\text {, }}$ When a rat that has undergone backward conditioning is presented with the CS, it reactivates the memory of the CS $\rightarrow$ context association, which indirectly reactivates the memory of the context $\rightarrow U S$ association and leads to activation of the hippocampus. $\mathbf{e}$, This indirect memory reactivation procedure can be used to induce destabilization of the context $\rightarrow$ US memory, such that it requires protein synthesis to persist in the brain. This can be blocked by rapamycin to attenuate subsequent fear.

for assessing the indirect reactivation of the memory trace and its neural basis.

Ressler and colleagues ${ }^{10}$ first established behaviorally that their hypothesized associative structure was correct by using extinction of the context to probe subsequent fearful behavior. While contextual extinction did not affect forward conditioning (as the cue is the better predictor of shock and overshadows the context), contextual extinction did impair backward conditioning (where the context is an essential component of the cue $\rightarrow$ context $\rightarrow$ shock association). Drawing on the wealth of evidence that implicates the dorsal hippocampus in the representation of contexts, they then compared recruitment of the hippocampus in the two fear-conditioning procedures, finding that backward conditioning particularly recruited the dentate gyrus. Using a viral-based approach to label the context memory engram with mCherry, they were able to show that subsequent presentation of the cue in a memory-reactivation session led to activation of the same mCherry-labeled engram, providing molecular evidence supporting the indirect reactivation of the memory trace. Furthermore, when they not only labeled with mCherry, but also tagged the context memory engram with an excitatory designer receptor exclusively activated by designer drug (DREADD), they found that they were able to produce conditioned freezing with administration of the DREADD agonist clozapine- $N$-oxide (CNO).

Their final experiment explicitly tested the hypothesis that indirect reactivation of the contextual fear memory-through re-exposure to the cue alone-would be sufficient to make the fear memory once again vulnerable to disruption. Specifically, the authors tested whether reconsolidation of the fear memory could be blocked with the protein synthesis inhibitor rapamycin. Consistent with the hypothesis that the forward fear memory does not recruit or require a contextual memory engram,
Ressler and colleagues found that the forward fear memory remained intact following the administration of rapamycin to the hippocampus in conjunction with cue re-exposure to reactivate the memory. (This is most likely due to the fact that another brain region critical for Pavlovian associations, the amygdala, can support reconsolidation of the cue-fear memory independently of the hippocampus.) By contrast, and importantly, rats that had undergone backward conditioning required protein synthesis within the hippocampus for the memory to reconsolidate and persist. When the contextual fear memory was indirectly reactivated through re-exposure to the cue, administration of rapamycin to the hippocampus attenuated subsequent fear. This suggests that it is possible to induce the reconsolidation of memories, even if the target memory is not directly reactivated.

The capacity to indirectly reactivate memories and render them susceptible to disruption overcomes a major 
potential hurdle in the translation of reconsolidation-based approaches to the clinic, where imaginal-exposure or virtual-reality approaches are far more tractable than direct re-exposure to trauma-related cues. Beyond practical issues-important as they are-this research also has theoretical implications. One major theory of PTSD suggests that trauma memories spread through a memory network, 'contaminating' other memories that were not originally associated with the trauma ${ }^{11}$. Even in 'simple' animal learning procedures like fear conditioning, it is known that a neural circuit supports fearful behavior, even if research has emphasized the importance of specific structures such as amygdala. Perhaps surprisingly, there has been little research investigating the impact of targeting a memory in one structure within a neural circuit on the representation of the memory in the other components of that circuit. Although Ressler et al. ${ }^{10}$ did not aim to address this question, it is straightforward to see how the approach they used could allow the impact of both direct and indirect memory reactivation on distributed engrams to be assessed.

Ultimately, as well as providing support for the translation of reconsolidationbased interventions for PTSD, Ressler and colleagues' findings ${ }^{10}$ act as a springboard for a host of future studies. It remains unknown whether older, more remote, or more extensively trained memories would also become unstable following indirect reactivation, which would be of great interest and importance considering the evidence supporting systems-level (re)consolidation and known boundary conditions for directly reactivated memories. Related to this, it is possible that other Pavlovian memories, such as cue-drug memories that promote relapse in addicted patients trying to remain abstinent, could also be indirectly reactivated; if so, that would extend the impact of the study to other mental health disorders. Beyond Pavlovian fear memories, it is not clear whether the instrumental memories that support action-outcome and habitual behavior-for example, active or passive avoidance for PTSD_-would share the same boundary conditions on reactivation (whether directly or indirectly reactivated). These are important questions that need to be addressed in the field of reconsolidation. What is very clear from the work of Ressler and colleagues ${ }^{10}$ is the impact and explanatory power afforded by using sophisticated behavioral approaches alongside cutting-edge neuroscience techniques. Careful and clever design of behavioral procedures is extremely powerful when it comes to understanding the outcome of sophisticated neural manipulations.

\section{Amy L. Milton (D) $₫$}

Department of Psychology, University of Cambridge, Cambridge, UK.

$\bigotimes_{\text {e-mail:alm46@cam.ac.uk }}$

Published online: 1 April 2021

https://doi.org/10.1038/s41593-021-00834-4

\section{References}

1. Ritchie, H. \& Roser, M. Mental health. Our World in Data https:// ourworldindata.org/mental-health (2018).

2. Chamberlain, S. R., Grant, J. E., Trender, W., Hellyer, P. \& Hampshire, A. BJPsych Open 7, e47 (2021).

3. American Psychiatric Association. The Diagnostic and Statistical Manual of Mental Disorders (5th ed.). (American Psychiatric Publishing, 2013)

4. Keane, T. M., Zimering, R. T. \& Caddell, R. T. Behav. Ther. 8, 9-12 (1985).

5. Craske, M. G., Treanor, M., Conway, C. C., Zbozinek, T. \& Vervliet, B. Behav. Res. Ther. 58, 10-23 (2014).

6. Holmes, E. A., Craske, M. G. \& Graybiel, A. M. Nature 511, 287-289 (2014).

7. Nader, K., Schafe, G. E. \& LeDoux, J. E. Nat. Rev. Neurol. 1, 216-219 (2000).

8. Suzuki, A. et al. J. Neurosci. 24, 4787-4795 (2004).

9. Dębiec, J., Doyère, V., Nader, K. \& Ledoux, J. E. Proc. Natl. Acad. Sci. USA 103, 3428-3433 (2006).

10. Ressler, R.L., Goode, T.D., Kim, S., Ramanathan, K.R. \& Maren, S. Covert capture and attenuation of a hippocampus-dependent fear memory. Nat. Neurosci. https://doi.org/10.1038/s41593-021 00825-5 (2021).

11. Foa, E. B., Steketee, G. \& Rothbaum, B. O. Behav. Ther. 20, 155-176 (1989).

Competing interests

The author declares no competing interests.

\section{From base pair to brain}

In new research, Smith et al. identify thousands of novel genetic associations with human brain structure and function, including those on the $X$ chromosome, by analyzing $4,000 \mathrm{MRI}$-derived traits measured in almost 40,000 individuals from the UK Biobank resource.

\section{Nana Matoba and Jason L. Stein}

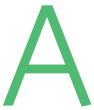
ny two humans have, on average, about four million genetic differences, or variants, from each other. A well-defined subset leads to inter-individual differences in outwardly observable traits like height, weight, and eye color, but which lead to changes in traits more relevant to the neuroscience field? Our knowledge of genetic differences influencing human brain structure and function is expanding through the measurement of macroscale human brain traits using in vivo neuroimaging methods such as MRI in large, genotyped populations. The great hope for this field is that by finding genetic risk factors associated with both neuropsychiatric disorders and with brain structure and function, causal mechanisms will also be found leading from the genome through molecular and cellular sequelae to gross brain structural changes, altered behavior, and risk for neuropsychiatric disorders. In other words, imaging genetics may identify brain regions, functions, or traits causally involved in complicated, behaviorally defined neuropsychiatric disorders. With knowledge about which regions and/or functions are responsible for these disorders, genetic variant function can be modeled in appropriately differentiated stem cell model systems or within relevant brain regions of animal models to experimentally manipulate and potentially reverse disorder-associated pathways.

Common genetic variants are older and have been refined by selective pressure 